\title{
Vulnerabilidades e ações desenvolvidas na população indígena de Tocantínia - TO acometida por COVID-19: Percepção dos profissionais da saúde
}

Vulnerabilities and actions developed in the indigenous population of Tocantínia - TO affected by

COVID-19: Perception of health professionals

Vulnerabilidades y acciones desarrolladas en la población indígena de Tocantínia - TO afectada

por COVID-19: Percepción de los profesionales de la salud

Recebido: 24/10/2021 | Revisado: 27/10/2021 | Aceito: 30/10/2021 | Publicado: 31/10/2021

\author{
Samara Silva de Sousa \\ ORCID: https://orcid.org/0000-0003-1954-5601 \\ Instituto Tocantinense Presidente Antônio Carlos, Brasil \\ E-mail: samysilva86@gmail.com \\ Andressa Layse Calixto Silva \\ ORCID: https://orcid.org/0000-0001-9157-9211 \\ Instituto Tocantinense Presidente Antônio Carlos, Brasil \\ E-mail: andressa.12lcs@gmail.com \\ Andressa de Sousa Brito \\ ORCID: https://orcid.org/0000-0002-6210-6666 \\ Instituto Tocantinense Presidente Antônio Carlos, Brasil \\ E-mail: andressa.desbrito@gmail.com \\ Alicia Leocádio Noleto \\ ORCID: https://orcid.org/0000-0002-2940-4784 \\ Instituto Tocantinense Presidente Antônio Carlos, Brasil \\ E-mail: alicianooleto@ hotmail.com \\ Pollyanna de Ulhôa Santos \\ ORCID: https://orcid.org/0000-0003-3916-6190 \\ Instituto Tocantinense Presidente Antônio Carlos, Brasil \\ E-mail: pollyannaulhoa@hotmail.com
}

\begin{abstract}
Resumo
Objetivo: Analisar a percepção dos profissionais de saúde sobre as vulnerabilidades dos povos indígenas diante do COVID-19, bem como identificar estratégias que são usadas para minimizá-las. Metodologia: Estudo do tipo qualitativo, descritivo, elaborado após a análise de questionários para avaliação do conhecimento e comportamento referido desses povos na visão dos profissionais que atuam diretamente e efetivamente nessa assistência. Os dados apresentados foram executados pelo software IRAMUTEQ com análises estatísticas sobre os corpus textuais e palavras. Foi aplicada a Classificação Hierárquica Descendente, em que as palavras foram aglomeradas em classes e os dados interpretados para a discussão subsequente. Resultados: Foram apresentadas três categorias: Estrutura física e Equipamentos de Proteção Individual (EPI's) para o atendimento de saúde aos indígenas; Ações de promoção de Saúde e prevenção de agravos referentes à COVID- 19 nas aldeias e a percepção dos indígenas sobre o coronavírus na visão dos profissionais. As categorias foram elaboradas de acordo com a frequência das palavras citadas nas entrevistas aos profissionais, conforme a Classificação Hierárquica Descendente (CHD). Considerações finais: Notouse o empenho dos profissionais de saúde na assessoria dos povos indígenas, assim como na propagação de informações e medidas de prevenção ao coronavírus. Apesar das dificuldades enfrentadas, como a resistência e os hábitos culturais, houveram grandes mudanças que contribuíram nas estratégias de promoção à saúde e prevenção de doenças.
\end{abstract}

Palavras-chave: COVID-19; Profissionais de saúde; Povos indígenas; Estratégias; Prevenção.

\begin{abstract}
Objective: To analyze the perception of health professionals about the vulnerabilities of indigenous peoples facing COVID-19, as well as to identify strategies to minimize them. Methods: This was a quantitative descriptive study, developed after the analysis of questionnaires to evaluate the knowledge and referred behavior of these peoples from the perspective of professionals that provide these services directly. The data presented were run by the software IRAMUTEQ with statistical analysis on the text corpus and words. The Descending Hierarchical Classification was applied, in which the words were agglomerated into classes and the data interpreted for subsequent discussion. Results: Three categories were presented: Physical structure and Personal Protective Equipment (PPE) used during care of indigenous people; Health promotion actions and prevention of diseases related to COVID-19 in the villages
\end{abstract}


and the perception of indigenous people about the coronavirus from the perspective of health care professionals. The categories were elaborated according to the frequency of the words mentioned in the interviews with the professionals, according to the Descending Hierarchical Classification (CHD). Final considerations: It was noted the commitment of health professionals in advising the indigenous people, as well as in spreading information and preventive measures against coronavirus. Despite the challenges presented, such as resistance and cultural habits, there were major changes that contributed to health promotion strategies and disease prevention.

Keywords: COVID-19; Allied health Personnel; Indigenous people; Health strategies; Disease prevention.

\section{Resumen}

Objetivo: Analizar la percepción de los profesionales de la salud sobre las vulnerabilidades de los pueblos indígenas ante el COVID-19, así como identificar las estrategias que se utilizan para minimizarlas. Métodos: Se trata de un estudio cualitativo, descriptivo, desarrollado después del análisis de los cuestionarios para evaluar el conocimiento y el comportamiento autoinformado de estas personas desde la perspectiva de los profesionales que trabajan directa y eficazmente en este cuidado. Los datos presentados fueron ejecutados por el software IRAMUTEQ con análisis estadístico del corpus textual y palabras. Se aplicó la clasificación Jerárquica Descendente, en que las palabras se agruparon en clases y los datos se interpretaron para su posterior discusión. Resultados: Se presentaron tres categorías: estructura física y Equipo de Proteccíon Personal (EPP) para la atención de salud de los pueblos indígenas; Acciones de promoción de la salud y prevención de enfermedades relacionadas con COVID-19 en los pueblos y La percepción de los indígenas sobre el coronavirus en la mirada de los profesionales. Las categorías se elaboraron según la frecuencia de las palabras mencionadas en las entrevistas con los profesionales, según el CHD. Consideraciones finales: Se destacó el compromiso de los profesionales de la salud en la asesoría a los pueblos indígenas, así como en la difusión de información y medidas para prevenir el coronavirus, a pesar de las dificultades enfrentadas, como resistencias y hábitos culturales, hubo cambios importantes que contribuyeron en las estrategias de promoción de la salud y prevención de enfermedades.

Palabras clave: COVID-19; Técnicos medios en salud; Pueblos indígenas; Estrategias; Prevención de enfermedades.

\section{Introdução}

Em dezembro de 2019, um novo vírus identificado como coronavírus causador da Síndrome Respiratória Aguda Grave (SARS), foi relatado na cidade de Wuhan, China, com uma rápida incidência de infecção que se espalhou no referido país e em todo o mundo. O Sars-CoV-2 mais conhecido como COVID-19 pertence à família Coronaviridae do gênero betacoronavirus. Sua transmissão é geralmente via gotículas aéreas para a mucosa nasal por meio de contato próximo entre pessoas, mãos ou superfícies contaminadas (Halaji, et al., 2020).

Estudos apontam que portadores de doenças crônicas, idosos, mulheres grávidas e pessoas com problemas respiratórios detém uma maior taxa de morbidade e mortalidade (Halaji, et al., 2020). Os sintomas podem variar desde um resfriado leve associado a tosse, febre, coriza, dor de garganta até manifestações mais graves como dificuldade para respirar, perda de olfato (anosmia), alteração do paladar (ageusia), dispneia, entre outros (Brasil, 2020).

Além dos sintomas típicos causados pelo Sars-CoV-2, doenças relacionadas à saúde mental têm ganhado um espaço considerável levando a preocupações de saúde pública. Em situações de pandemia, os indivíduos sentem-se preocupados e estressados, especialmente as populações mais vulneráveis e os profissionais de serviços essenciais. O medo, o estresse e as incertezas podem levar a consequências em longo prazo, acarretando problemas relacionados à saúde mental. Esses problemas podem surgir em vista do temor da exposição e do contágio, do confinamento e do isolamento, e pelas medidas de quarentena, que implicam em mudanças na rotina de vida das pessoas (Lobo \& Rieth, 2021).

Até o mês de outubro de 2021, na última atualização do Ministério da Saúde, houveram no Brasil 21.711.843 casos confirmados, 20.885 .484 casos recuperados e 605.139 mortes pelo vírus Sars-CoV-2. Atualmente, o país possui um plano nacional de operacionalização de vacinação contra a COVID-19 que segundo o Ministério da Saúde é uma medida adicional de resposta ao enfrentamento da doença nomeada Emergência de Saúde Pública de Importância Internacional (ESPII) que propõe ações de vacinação nos três níveis de gestão (Brasil, 2021).

Segundo a Secretaria Especial de Saúde Indígena (SESAI), criada em 2010, há pelo menos 416 etnias indígenas diferentes no Brasil. Ainda, de acordo com a Fundação Nacional do Índio (FUNAI), o último censo reportado pelo Instituto 
Brasileiro de Geografia e Estatística (IBGE) registrou que há cerca de 817.963 indígenas distribuídos por todo o território nacional. Dessa forma, estima-se que os índios estão presentes em todos os estados brasileiros, sendo que a região Norte concentra o maior número desses povos, principalmente no Estado do Amazonas (Cupertino, et al., 2020). De acordo com o Ministério da Saúde (2020), os povos indígenas possuem uma maior vulnerabilidade biológica a viroses, com destaques às infecções respiratórias. Dessa forma, observou-se ao longo do tempo que as epidemias e os índices elevados de mortalidade por doenças respiratórias tiveram papel considerável na redução da população indígena no Brasil, sendo que as doenças do trato respiratório continuam como a maior causa de mortalidade infantil na população indígena. Devido a esses fatores, ressalta-se que a pandemia de COVID-19 trouxe bastante preocupação sobre a população indígena no Brasil, devido à alta mortalidade histórica por doenças infecto parasitárias (Cupertino, et al., 2020).

Desse modo, o índice de vulnerabilidade assume valores mais elevados quando comparado à população geral. Além disso, alguns comportamentos e aspectos culturais influenciam diretamente a disseminação do vírus nessas comunidades. O compartilhamento de cabaças e outros utensílios domésticos, bem como habitação comunitária influenciam nas estratégias que devem ser ressaltadas a fim de mitigar os impactos gerados pela pandemia do SARS-Cov-2 (De León-Martínez, et al., 2020).

De acordo com o boletim epidemiológico da SESAI, o primeiro caso de COVID-19 em indígenas na região Norte foi confirmado no dia 01 de abril de 2020 na região do Alto Rio Solimões, no estado do Amazonas e a primeira morte data de 10 de abril de 2020 no povo Yanomami, também no estado do Amazonas. Desde então os casos cresceram, e de acordo com os dados de outubro de 2021 já são 54465 casos confirmados e 831 óbitos (Brasil, 2021). Dessa forma, a COVID-19 veio para somar em uma lista de ameaças à saúde pública e segurança já enfrentadas pelas populações rurais e indígenas (Castro, et al., 2020). Além disso, a vulnerabilidade desse povo inclui também questões geográficas e estruturais devido ao distanciamento de centros hospitalares capazes de suprir a necessidade de saúde dos indígenas afetados pelo SARVSCov-2. O presente artigo visa compreender como a COVID-19 impactou as populações indígenas da região de Tocantínia- TO em suas diversas particularidades e identificar medidas que foram usadas para amenizar as dificuldades vivenciadas por esses povos, na perspectiva de profissionais de saúde que acompanham as tribos.

\section{Metodologia}

O presente estudo seguiu os princípios éticos e legais que regem a pesquisa científica, que estão descritos na Resolução no 466/2012 do Conselho Nacional de Saúde. O mesmo foi submetido e aprovado pelo Comitê de Ética e Pesquisa (CEP) do Centro Universitário Tocantinense Presidente Antônio Carlos (UNITPAC), de acordo com o parecer número: 4.943.539 - CAAE 47815420.1.0000.0014, após o cadastro prévio na Plataforma Brasil.

Foi realizado um estudo do tipo qualitativo, descritivo, que apresenta aspectos da realidade, direcionando-o para compreensão e explicação da dinâmica das relações sociais (Gerhardt \& Silveira, 2009) com caracterização da amostra e com base na análise de conteúdo de Bardin.

Os dados foram coletados através do banco de dados da secretaria especial de saúde indígena (SESAI), de entrevista feita aos profissionais de saúde que atuam nas tribos indígenas da região de Tocantínia - TO por meio da Plataforma Google Meet e do instrumento Questionário para avaliação do conhecimento e comportamento referido dos trabalhadores sobre precauções padrão, que foi aplicado utilizando o Google Forms. Foi realizada a coleta de dados, por meio de categorização das entrevistas realizadas entre Setembro e Outubro de 2021, descrevendo o perfil dos profissionais com as variáveis: sexo, categoria profissional e tempo de atuação na região indígena.

As informações levantadas após a transcrição foram armazenadas e analisadas através do software IRAMUTEQ (Interface de R pour les Analyses Multidimensionnelles de Textes et de Questionnaires), o mesmo é gratuito, de fonte aberta, e permite fazer análises estatísticas sobre os corpus textuais e palavras. Foi utilizada a Classificação Hierárquica Descendente 
(CHD), responsável por aglomerar palavras em classes, sendo cada classe composta por um vocabulário semelhante entre si e, ao mesmo tempo, destoando daqueles de outras classes. Dessa forma, os segmentos de texto são classificados em virtude dos respectivos vocabulários.

Pautando-se no conteúdo das entrevistas e da CHD gerada pelo IRAMUTEQ, foram criadas as categorias de análise, seguindo as etapas propostas pela técnica de análise de conteúdo temática de Bardin. Para isso, as entrevistas foram submetidas a pré-análise mediante a transcrição dos depoimentos; em seguida, foi realizada a explanação do material, para obter o recorte temático, e em seguida a interpretação dos resultados para aprimorar a compreensão das categorias de análise para discussão. Participaram da pesquisa, quatro enfermeiros, sendo uma responsável técnica pelas equipes de saúde que atuam nas tribos indígenas da região de Tocantínia (TO) e dois cirurgiões-dentistas.

\section{Resultados}

Dentre os 125 profissionais de saúde que atuam nas aldeias do território de Tocantínia (Tabela 1), foram incluídos na pesquisa os que atuam nas categorias de nível superior. Dos onze profissionais que atendiam aos critérios de inclusão e receberam o convite para participar da pesquisa, seis aceitaram e foram entrevistados.

Tabela 1 - Caracterização da quantidade de profissionais de saúde atuantes nas aldeias do território de Tocantínia - TO.

\begin{tabular}{lc}
\hline Profissionais de saúde & Quantidade \\
\hline Médicos & 3 \\
Enfermeiros & 6 \\
Agentes indígenas de saúde & 21 \\
Técnicos de enfermagem & 22 \\
Odontólogo & 2 \\
Motorista & 10 \\
Auxiliar de saúde bucal & 1 \\
Assistente administrativo & 1 \\
Agentes indígenas de saneamento & 59 \\
Total & $\mathbf{1 2 5}$ \\
\hline
\end{tabular}

Fonte: Autores.

Em relação à análise realizada pelo IRAMUTEQ, foram encontradas 2966 ocorrências de palavras, 556 formas ativas, sendo analisados 84 segmentos de texto pelo software, com 64,29\% de retenção na Classificação Hierárquica Descendente (CHD).

A CHD dividiu o corpus em cinco classes, sendo três delas aglomeradas com a mesma temática. Com base na organização, análise e conteúdo das transcrições das entrevistas, foram identificadas como categorias: Estrutura física e Equipamentos de Proteção Individual (EPI’s) para o atendimento de saúde aos indígenas; Ações de promoção de Saúde e prevenção de agravos referentes à COVID- 19 nas aldeias; A percepção dos indígenas sobre o coronavírus na visão dos profissionais. As categorias foram elaboradas de acordo com a frequência das palavras citadas nas entrevistas aos profissionais, conforme a CHD. 


\section{Estrutura física e EPI's para o atendimento de saúde aos indígenas}

Os profissionais afirmaram que não possuem uma estrutura física adequada para a realização dos atendimentos nas aldeias da região de Tocantínia, pois destas apenas uma contém Unidade Básica de Saúde (UBS) em seu território.

"Sobre a estrutura física que eu disponho para trabalhar, só tem unidade na aldeia Funil que é a aldeia maior. As outras atendo debaixo de árvores, instalações em casas de alguém, chapéus de palha, tentando sempre procurar uma sombra. Quando chove tem que atender na casa de alguém. A estrutura é um pouco precária em algumas aldeias, por isso é mais difícil. Às vezes, não conseguimos oferecer um atendimento de qualidade porque não tem estrutura." (Entrevista 6 - Enfermeiro)

"Na maioria das vezes o atendimento acontece nas aldeias na casa dos indígenas onde é improvisado uma estrutura com bancos de madeira embaixo de árvores para fazer os atendimentos." (Entrevista 4- Enfermeiro)

Os procedimentos são realizados embaixo de árvores, nas grandes casas das aldeias ou na casa do cacique. Em relação aos atendimentos odontológicos, as cirurgiãs dentistas relataram que possuem apenas um "Odontomóvel" para atender todas as aldeias, por isso, só dispõem desse veículo uma vez ao mês.

"Não possui estrutura física em todas as aldeias, muitas aldeias fazem o atendimento onde der, embaixo de árvores, na casa do cacique, em casas grandes. Eles possuem o "OdontoMóvel" só que é apenas um para todas as aldeias, então geralmente conseguem atender 1 vez ao mês com ele. Em algumas aldeias possui uma estrutura física para o atendimento como a UBS." (Entrevista 1 - Cirurgião dentista)

Além disso, como observado no grupo de palavras da Classe 5, os profissionais de saúde relataram que a falta inicial de EPIs essenciais como máscara e luvas não impactaram significativamente no trabalho exercido, pois sempre foram amparados com os devidos materiais para assistência das aldeias por órgão como o Distrito Sanitário Especial Indígena (DSEI).

"Em relação aos EPIs já houve momentos de falta e nesses casos conseguimos pegar no município." (Entrevista 5Enfermeiro)

"Dispomos do suporte de uma UBS de Tocantínia, do hospital de referência e do CAPS de Miracema, mas na maioria das vezes o atendimento acontece nas aldeias, na casa dos indígenas onde é improvisado um logo o DSEI repôs esse material." (Entrevista 4- Enfermeiro)

\section{Ações de promoção de Saúde e prevenção de agravos referentes à COVID- 19 nas aldeias}

De acordo com o relato dos profissionais de saúde, todas as aldeias do território de Tocantínia receberam orientações sobre as medidas de prevenção contra a COVID-19. Dentre as medidas mencionadas, destacam- se: cartilhas na linguagem própria dos Xerentes, panfletos, rodas de conversa e orientações verbais que relacionam- se com as palavras descritas na classe 2.

"Eram feitas rodas de conversas, palestras sem público grande, mas com caciques e líderes que pudessem passar as informações para o resto da aldeia, orientações verbais, distribuições de kits.” (Entrevista 4- Enfermeiro)

"Fazemos visitas domiciliares casa por casa, passamos as orientações, mostramos a forma correta de higienização, entregamos alguns panfletos e principalmente a comunicação com os agentes de saúde, técnicos de enfermagem, e os auxiliares que são indigenas. O que tornava o processo de propagação e admissão mais fácil." (Entrevista 2Cirurgião- dentista) 
“Verbalmente, através de visitas domiciliares, panfletos, cartilhas (DSEI), cartilhas na lingua deles.” (Entrevista 5 Enfermeiro)

Apesar das orientações de prevenção terem sido amplamente repassadas pelos profissionais de saúde, foi observada uma certa resistência por parte dos indígenas em aderi-las devido a sua forma de moradia e organização familiar.

"Além de todas as orientações de distanciamento social que receberam, foi muito difícil para eles pois vivem muito unidos, muitos vivem na mesma casa." (Entrevista 6- Enfermeiro)

“As informações chegaram, só que eles não aceitaram bem, pois é cultura deles essa questão do convívio social e viver todos na mesma casa. Eles continuaram com a vida normal, a única coisa que deixaram foram os eventos, mas agora já voltaram." (Entrevista 5- Enfermeiro)

"Os pacientes atendidos recebiam as devidas orientações e informações, mas tinham muita resistência na execução dessas medidas." (Entrevista 2- Cirurgião-dentista)

\section{A percepção dos indígenas sobre o coronavírus na visão dos profissionais}

Segundo os profissionais de saúde, no início da pandemia houve baixa adesão das medidas de prevenção e muita resistência e descrença a respeito da gravidade da doença, principalmente devido à disseminação de falsas notícias.

"No início da pandemia muitos não acreditavam na doença, comparavam o coronavírus com uma gripezinha, contudo, outros pareciam ansiosos e nervosos. Em relação à negação ao coronavírus, tivemos que implantar um sistema de educação, pois todos são muito unidos e as casas não possuem muros e dessa forma é muito difícil manter o distanciamento social." (Entrevista 1 - Cirurgião- dentista)

“Observamos um sentimento de negação, a maioria tem acesso à internet, TV, mas não acreditavam. Eles negaram muito, não sabiam da real dimensão do problema." (Entrevista 3-Enfermeiro)

"Foi montada uma equipe de estratégia e foi muito difícil no início. Fomos impedidos de entrar em algumas aldeias, pois diziam que os profissionais levariam a COVID para as aldeias. Tivemos dificuldade em relação à vacina $e$ demorou para ter uma aceitação geral. A primeira impressão das informações da TV ficou muito forte." (Entrevista 5- Enfermeiro)

"Foi bastante complicado pois houve muita fake news, então eles ficaram com muito medo, apreensivos. Não queriam se vacinar por medo. Aderiram bastante os medicamentos naturais para prevenção de covid como garrafadas. Os profissionais orientavam que eles evitassem visitar a cidade, mas sempre tem aqueles que não obedeciam." (Entrevista 6- Enfermeiro)

Apesar da objeção inicial, ao longo do tempo houve uma mudança nesse comportamento como expressa os dados gerados pelo IRAMUTEQ, nas classes 1, 3 e 4 da CHD em que foi observado que o sentimento de medo e preocupação foi se instalando, especialmente após o surgimento de casos de COVID-19 e morte nas aldeias e, nesse contexto, as medidas passaram a serem cumpridas com mais vigor. 
“Ao longo do tempo observamos mudanças nesse comportamento e percepção por uma grande maioria deles, tanto em relação à doença quanto pela vacina. Eles tinham muito receio de ir ao hospital, pois associavam a hospitalização à morte, mas hoje já estão bem menos resistentes, com a ajuda dos profissionais que os orientaram de forma adequada." (Entrevista 1- Cirurgião- dentista)

“Eles aos poucos aceitaram que a COVID infelizmente estava no nosso meio, adotaram o modo de prevenção tanto do branco como dos indígenas, usaram muitas ervas, garrafadas, remédios caseiros. Adotaram a higienização das mãos, usavam máscara frequentemente, porém os mais velhos eram mais resistentes." (Entrevista 4- Enfermeiro)

“Sim, a percepção deles mudou totalmente. Há pouco tempo que chegou nos Xerente, foi a etnia com menos contaminação. Aqueles que não aceitavam a vacinação agora estão aceitando." (Entrevista 5- Enfermeiro)

"A partir do momento que alguns começaram a ficar infectados eles começaram a ficar preocupados. Então começaram a colocar cercas para evitar a entrada de pessoas não indígenas, somente os profissionais da saúde podiam entrar." (Entrevista 6- Enfermeiro)

\section{Discussão}

O aparecimento de um novo vírus de fácil contágio como o Sars-CoV-2 acarretou grandes mudanças no serviço de saúde pública. Diante disso, profissionais de saúde se viram em uma situação de pânico e sobrecarga de trabalho e a população com sentimentos de medo e angústia. Nessa perspectiva, estudos apontam que houve alterações da saúde mental como depressão, ansiedade e estresse pelo medo de ser infectado por um patógeno novo pouco conhecido e potencialmente fatal (Schmidt, 2020; Asmundson, 2020; Carvalho, 2020; Wang, 2020; Zhang, 2020).

Ao longo dos anos epidemias do tipo parasitárias e infecciosas têm sido tragédias que acometem não só a população em geral, mas também etnias de culturas diversificadas. Nesse sentido, a comunidade indígena brasileira, desde a colonização do Brasil, em 1500, já passou por diversos períodos de adaptação a uma nova doença, como os eventos atuais da COVID-19. Ademais, esse histórico de contato com doenças infecciosas demonstrou que elas costumam se disseminar em grande velocidade. (Santos, 2020; ABRASCO, 2020)

Os povos indígenas possuem uma rede de atendimento à saúde com estruturas limitadas que apresentam desafios logísticos, geográficos, trabalhistas e pessoais. Dessa forma, frente a uma pandemia, novas dificuldades surgiram ao longo dos dias e acarretaram uma série de estratégias e ações a serem executadas a essa população. Não muito diferente dos centros hospitalares, os profissionais também enfrentaram dificuldades como a falta de estrutura para os atendimentos e, além disso, a resistência à prevenção da disseminação do vírus, devido a fortes hábitos culturais, que influenciaram na baixa adesão dessa população.

Na percepção dos profissionais responsáveis pela saúde indígena tal cenário causou um certo temor e angústia a essa população, em vista da mudança de costumes e atitudes tão inerentes à sua cultura. Sabe-se que nessa cultura, as habitações frequentemente têm grande número de pessoas, reuniões e festas fazem parte de hábitos diários, além da produção e venda de artesanatos em locais públicos. Dessa forma, frente à pandemia todos esses costumes se tornaram restritos, culminando em mudanças psicológicas e dificuldade de aceitação da doença por parte desses povos (Abrasco, 2020; Ribeiro \& Rossi, 2020).

A mídia tradicional e as redes sociais são os principais instrumentos de divulgação de informações sobre a pandemia do coronavírus (Bazán et al, 2020). Devido a isso, sua fácil acessibilidade e riqueza de informações facilitou que as "fake news" em certo momento tivessem um impacto negativo nas estratégias de prevenção da COVID-19. Isso influenciou a população geral e os povos indígenas a desacreditarem na gravidade da doença e como consequência não aderirem tão bem as 
orientações que lhe foram passadas inicialmente (Neto et al., 2020). Algumas pessoas acreditaram em informações que o novo vírus e a vacina seriam instrumentos criados para extermínio da população indígena. Reafirmando o que diz Garnelo (2011), quando há o desencontro entre a ideologia cultural dos indígenas e as ideologias biomédicas, há uma falha no processo do cuidado de saúde.

O uso de EPI 's, como a máscara facial, é uma das principais medidas de prevenção da contaminação pelo coronavírus, além da higienização das mãos e o distanciamento social. O não uso de máscaras aliado às condições de moradia são fatores que predispõem à contaminação e propagação da Covid-19 entre os indígenas. Segundo os relatos apurados nas entrevistas, dentro da comunidade indígena o uso das máscaras não se cumpria e esse uso só acontecia quando havia deslocamento do índio para áreas urbanas. Além disso, em relação às medidas preventivas, o modo de habitação se tornou um grande impasse para que o distanciamento social ocorresse de forma efetiva (Abrasco, 2020).

A partir do momento em que tiveram uma percepção sobre o real perigo desse novo vírus, os indígenas tomaram medidas que protegessem o seu próprio povo e continuaram a tratar seus doentes com seus remédios naturais, como afirma a The Lancet Diabetes \& Endocrinology (2020): "O mundo tem muito a aprender com os povos indígenas — desde como viver harmoniosamente e de forma sustentável com o planeta até seu conhecimento de medicamentos tradicionais." Assim, os profissionais entrevistados, acreditam que o uso de medicamentos naturais e o cumprimento das orientações dadas por eles e pelos demais profissionais de saúde, pôde ser uma estratégia eficaz para conter a disseminação de doenças infectocontagiosas como a Covid-19 e minimizar os impactos e consequências causadas por essa doença. Para isso, é imprescindível que os profissionais tenham uma relação dialógica com os caciques, pois estes possuem autoridade para viabilizar o cumprimento das medidas de contenção dentro das aldeias (Ribeiro \& Rossi, 2020).

Foi observado que os indígenas têm uma boa percepção a respeito dos profissionais de saúde, visto que eles além de garantirem uma assistência especializada, também são responsáveis pela divulgação das informações e orientações necessárias para a prevenção de agravos e doenças. Apesar da cultura da pajelança, as equipes de saúde têm um espaço garantido nessas aldeias e realizam as ações sem resistência. Notou-se, também, que a articulação do trabalho desses profissionais com os agentes indígenas de saúde (AIS) próprios das comunidades indígenas da região foi de extrema importância para que as orientações fossem disseminadas e compreendidas da melhor forma pela população.

\section{Conclusão}

Com os dados analisados nas entrevistas foi possível inferir a percepção dos profissionais que atuam nas aldeias da região de Tocantínia. Nesse sentido, foi observado o empenho dos profissionais e AISs locais em garantir que toda a população indígena conhecesse e colocasse em prática as medidas de prevenção ao coronavírus, contudo, houve uma grande resistência à aceitação da nova doença e adesão dos indígenas às medidas de contenção, principalmente devido aos seus hábitos culturais de viver em grupos e a repercussão de "fake news". No entanto, apesar do início conturbado, com o passar dos meses houve uma mudança de comportamento e eles mesmos foram os agentes de combate ao coronavírus.

Esse estudo trouxe, assim, uma maior visibilidade a esses povos, suas dificuldades e aspectos culturais, possibilitando, dessa forma, uma melhor compreensão da realidade vivida, bem como uma percepção mais detalhada dos profissionais de saúde quanto às variáveis do processo de assistência e às ações que são utilizadas para mitigar os obstáculos encontrados por essas equipes.

Ademais, recomenda-se que mais estudos sejam realizados sobre a saúde dos indígenas, sobretudo no contexto da COVID-19, buscando identificar mais profundamente outras vulnerabilidades desses povos, como por exemplo, as alterações em níveis psicológicos. 


\section{Referências}

ABRASCO (2020). A COVID-19 e os povos indígenas: desafios e medidas para controle do seu avanço. Rio de Janeiro, 21 de março de 2020. Website da Associação Brasileira de Saúde Coletiva.

Asmundson, G. J. G., \& Taylor, S. (2020). Coronaphobia: fear and the 2019-nCoV outbreak. Journal of Anxiety Disorders. 70 , 102196.

Bazán, P. R., Azevedo Neto, R. M., Dias, J. A., Salvatierra, V. G., Sanches, L. G., Lacerda, S. S. et al. (2020). Exposição às informações sobre COVID-19 em mídias digitais e suas implicações para funcionários do setor de saúde: resultados de uma pesquisa on-line. einstein. 18: eAO6127. http://dx.doi.org/10.31744/ einstein_journal/2020AO6127

Brasil. (2020). Ministério da Saúde. O que é a Covid-19? https://www.gov.br/saude/pt-br/coronavirus/o-que-e-o-coronavirus

Brasil. (2021). Ministério da Saúde. Boletim Epidemiológico da SESAI. http://www.saudeindigena.net.br/coronavirus/mapaEp.php

Carvalho, P. M. M., Moreira, M. M., Oliveira, M. N. A., Landim, J. M. M., \& Rolim Neto, M. L. (2020). The psychiatric impact of the novel coronavirus outbreak. Psychiatry research, 286: 112902. https://doi.org/10.1016/j.psychres.2020.112902

Castro F., Lopes, G. R., \& Brondizio, E. S. (2020). The Brazilian Amazon in Times of COVID-19: from crisis to transformation?. Ambiente \& Sociedade. 23, 1-11. https://doi.org/10.1590/1809-4422asoc20200123vu2020L3ID

Cupertino, G. A., Cupertino, M. C., Gomes, A. P., Braga., L. M., \& Batista., R. S. (2020). COVID-19 and Brazilian Indigenous Populations. The American journal of tropical medicine and hygiene. 103 (2). 609- 612. https://doi.org/10.4269/ajtmh.20-0563

De León-Martínez, L. D. et al. (2020). Critical review of social, environmental and health risk factors in the Mexican indigenous population and their capacity to respond to the COVID-19. Science of The Total Environment. 733. 1-6. https://doi.org/10.1016/j.scitotenv.2020.139357

Garnelo, L. (2011). Aspectos socioculturais de vacinação em área indígena. História, Ciências, Saúde - Manguinhos, Rio de Janeiro, 18 (1). 175-190. https://doi.org/10.1590/S0104-59702011000100011

Gerhardt, T. E., \& Silveira, D. T. (2009). A pesquisa científica Métodos de Pesquisa. Editora da UFRGS.

Halaji, M. (2020). Emerging coronaviruses: first SARS, second MERS and third SARS-CoV-2. Epidemiological updates of COVID-19. La Infezioni in Medicina. 28, 6-17.

IBGE (2010). Censo demográfico 2010: Características gerais dos indígenas. Censo demogr., 1- 245.

Lobo, L. A. C., \& Rieth, C. E. (2021). Saúde mental e Covid-19: uma revisão integrativa da literatura. Saúde em debate, 45 (130), 885-901. https://doi.org/10.1590/0103-1104202113024

Neto, M., Gomes, T de O., Porto F. R., Rafael, R de M. R., Fonseca, M. H. S., \& Nascimento, J. (2020). Fake news no cenário da pandemia de Covid-19. Cogitare enferm. 25: e72627 http://dx.doi.org/10.5380/ce.v25i0.72627

Ribeiro, A. A., \& Rossi, L. A. (2020). Covid-19 pandemic and the motivations for demanding health service in indigenous villages. Rev. Bras. Enferm. 73 (6), 1- 6. 73(Suppl 2):e20200312. doi: http://dx.doi.org/10.1590/0034-7167-2020-0312

Santos, R. V., Pontes, A. L., \& Coimbra Junior, C. E. A. (2020). Um "fato social total”: covid-19 e povos indígenas no brasil. Cadernos de Saúde Pública, 36 (10), 1-5. http://dx.doi.org/10.1590/0102-311x00268220

Schmidt, B., Creapldi, M. C., Bolze, S. D. A., Neiva - Silva, L., \& Demenech, L. M. (2020). Saúde mental e intervenções psicológicas diante da pandemia do novo coronavírus (COVID-19). Estud. Psicol. 37: e200063. https://doi.org/10.1590/1982-0275202037e200063

The Lancet Diabetes \& Endocrinology. (2020). Indigenous peoples: resilience in the face of adversity https://www.thelancet.com/journals/landia/article/PIIS2213-8587(20)30273-4/fulltext

Wang, C., Pan, R., Wan, X., Tan, Y., Xu, L., Ho, C. S. et al. (2020). Immediate psychological responses and associated factors during the initial stage of the 2019 coronavirus disease (COVID-19) epidemic among the general population in China. Int J Environ Res Public Health. 17 , 1729. https://doi.org/10.3390/ijerph17051729

Zhang, C., Yang, L., Liu, S., Ma, S., Wang, Y., Cai, Z. et al. (2020). Survey of insomnia and related social psychological factors among medical staffs involved with the 2019 novel coronavirus disease outbreak. Frontiers in Psychiatry. 11, 1-9. https://doi.org/10.3389/fpsyt.2020.00306 